\title{
Argumentation-Based Paraconsistent Logics
}

\author{
Jonathan Ben-Naim \\ IRIT - CNRS \\ 118 , route de Narbonne \\ 31062, Toulouse Cedex 09, France
}

\begin{abstract}
Argumentation is a promising approach for reasoning with inconsistent information. Starting from a knowledge base encoded in a logical language, an argumentation system defines arguments and attacks between them using the consequence operator associated with the language. Finally, it uses a semantics for evaluating the arguments. The plausible conclusions to be drawn from the knowledge base are those supported by "good" arguments.

In this paper, we discuss two families of such systems: the family using extension semantics and the one using ranking semantics. We discuss the outcomes of both families and compare them.
\end{abstract}

\section{Introduction}

A paraconsistent logic consists of a language and a consequence operator returning rational conclusions even from inconsistent premises. Possibly, a paraconsistent logic may attach absolute or relative weights to the conclusions.

Note that inconsistency may be present in knowledge bases for mainly three reasons: i) a knowledge base may contain a default rule [10] which has exceptions and the former leads to an opposite conclusion than the latter; ii) in model-based diagnosis [9], the description of the normal behavior of a system may be conflicting with the observations made on this system; iii) an inconsistent knowledge base may result from the union of several consistent knowledge bases pertaining to the same domain [5]. Whatever the source of inconsistency, a paraconsistent logic is needed to deal with it.

As a consequence, there has been much work on constructing and investigating such logics. Two families can be distinguished: those that restore consistency (e.g., [10,11]) and those that tolerate inconsistency without exploding (e.g., $[4,6])$. One important instance of the first family computes the maximal (for set inclusion) consistent subbases of a knowledge base, then chooses the conclusions that follow from all those subbases. Regarding the second family, a prominent approach considers many valued interpretations with the crucial particularity that they can be models of even inconsistent premises and thus can be used to draw conclusions.

Since early nineties, due to its explanatory power, argumentation has become a promising approach for handling inconsistency. An argumentation system consists of arguments, attacks between them and a semantics for evaluating the arguments. The latter are built from a knowledge base encoded in a particular language and using the consequence operator associated with the language. The attacks generally refer to the inconsistency of the base. Finally, the rational conclusions induced by such an argumentation system are those supported by acceptable arguments wrt the semantics. 
In this paper, we recall the two families of semantics developed in the literature, namely extension semantics introduced by Dung in [8] and ranking semantics defined more recently in [1]. We discuss and compare the paraconsistent logics induced by each family. We show that logics based on extension semantics return flat conclusions and restore consistency while those based on ranking semantics return ranked conclusions and tolerate inconsistency.

\section{Argumentation Systems}

Argumentation systems are built on an underlying monotonic logic. In this paper, we focus on Tarski's monotonic logics [12]. Indeed, we consider logics $(\mathcal{L}, \mathrm{CN})$ where $\mathcal{L}$ is a set of well-formed formulas and $\mathrm{CN}$ is a consequence operator. It is a function from $2^{\mathcal{L}}$ to $2^{\mathcal{L}}$ which returns the set of formulas that are logical consequences of another set of formulas according to the logic in question. It satisfies the following basic properties:

1. $X \subseteq \mathrm{CN}(X)$

(Expansion)

2. $\mathrm{CN}(\mathrm{CN}(X))=\mathrm{CN}(X)$ (Idempotence)

3. $\operatorname{CN}(X)=\bigcup_{Y \subset_{f} X} \operatorname{CN}(Y)^{1}$ (Compactness)

4. $\operatorname{CN}(\{x\})=\mathcal{L}$ for some $x \in \mathcal{L}$

(Absurdity)

5. $\operatorname{CN}(\emptyset) \neq \mathcal{L}$ (Coherence)

A CN that satisfies the above properties is monotonic. The associated notion of consistency is defined as follows:

Definition 1 (Consistency). A set $X \subseteq \mathcal{L}$ is consistent wrt a logic $(\mathcal{L}, \mathrm{CN})$ iff $\mathrm{CN}(X) \neq$ $\mathcal{L}$. It is inconsistent otherwise.

Arguments are built from a knowledge base $\Sigma \subseteq \mathcal{L}$ as follows:

Definition 2 (Argument). Let $\Sigma$ be a knowledge base. An argument is a pair $(X, x)$ s.t. 1) $X \subseteq \Sigma$, 2) $X$ is consistent, 3) $x \in \mathrm{CN}(X)$ and $X$ is minimal (for set inclusion) wrt 1), 2) and 3).

Notations: Supp and Conc denote respectively the support $X$ and the conclusion $x$ of an argument $(X, x)$. For all $\mathcal{S} \subseteq \Sigma, \operatorname{Arg}(\mathcal{S})$ denotes the set of all arguments that can be built from $\mathcal{S}$ by means of Definition 2, Sub is a function that returns all the sub-arguments of a given argument. For all $\mathcal{E} \subseteq \operatorname{Arg}(\Sigma), \operatorname{Base}(\mathcal{E})=\bigcup_{a \in \mathcal{E}} \operatorname{Supp}(a)$. $\operatorname{Max}(\Sigma)$ is the set of all maximal (for set inclusion) consistent subbases of $\Sigma$.

An argumentation system is defined as follows.

Definition 3. An argumentation system $(A S)$ over a knowledge base $\Sigma$ is a pair $\mathcal{T}=$ $(\operatorname{Arg}(\Sigma), \mathcal{R})$ where $\mathcal{R} \subseteq \operatorname{Arg}(\Sigma) \times \operatorname{Arg}(\Sigma)$ is an attack relation such that for all $a, b \in \operatorname{Arg}(\Sigma)$, if $(a, b) \in \mathcal{R}$, then $\operatorname{Supp}(a) \cup \operatorname{Supp}(b)$ is inconsistent.

Note that $(a, b) \in \mathcal{R}$ (or $a \mathcal{R} b$ ) means that $a$ attacks $b$. It is also worth mentioning that the set $\operatorname{Arg}(\Sigma)$ may be infinite even when the base $\Sigma$ is finite.

\footnotetext{
${ }^{1} Y \subseteq f X$ means that $Y$ is a finite subset of $X$.
} 


\section{Logics Induced by Extension Semantics}

The most popular semantics were proposed by Dung in his seminal paper [8]. Those semantics as well as their refinements (e.g. in [3,7]) partition the powerset of the set of arguments into two classes: extensions and non-extensions. Every extension represents a coherent point of view. We illustrate the kind of paraconsistent logics induced by such semantics on the most popular ones, namely naive, stable and preferred. Before giving the formal definitions of the three semantics, we first introduce two key concepts on which they are based.

Definition 4 (Conflict-freeness-Defence). Let $\mathcal{T}=(\mathcal{A}, \mathcal{R})$ be an argumentation system, $\mathcal{E} \subseteq \mathcal{A}$ and $a \in \mathcal{A}$.

- $\mathcal{E}$ is conflict-free iff $\nexists a, b \in \mathcal{E}$ such that $a \mathcal{R} b$.

- $\mathcal{E}$ defends an argument a iff $\forall b \in \mathcal{A}$ such that $b \mathcal{R} a, \exists c \in \mathcal{E}$ such that $c \mathcal{R} b$.

Definition 5 (Semantics). Let $\mathcal{T}=(\mathcal{A}, \mathcal{R})$ be an argumentation system and $\mathcal{E} \subseteq \mathcal{A}$.

- $\mathcal{E}$ is a naive extension iff it is a maximal (w.r.t. set $\subseteq$ ) conflict-free set.

$-\mathcal{E}$ is a preferred extension iff it is a maximal (w.r.t. set $\subseteq$ ) set that is conflict-free and defends its elements.

- $\mathcal{E}$ is a stable extension iff it is conflict-free and attacks any argument in $\mathcal{A} \backslash \mathcal{E}$.

Notations: $\operatorname{Ext}_{x}(\mathcal{T})$ denotes the set of all extensions of $\mathcal{T}$ under semantics $x$ where $x \in\{n, p, s\}$ and $n$ (resp. $p$ and $s$ ) stands for naive (respectively preferred and stable). When we do not need to refer to a particular semantics, we write $\operatorname{Ext}(\mathcal{H})(\mathcal{T})$ for short.

Example 1. The argumentation system depicted below

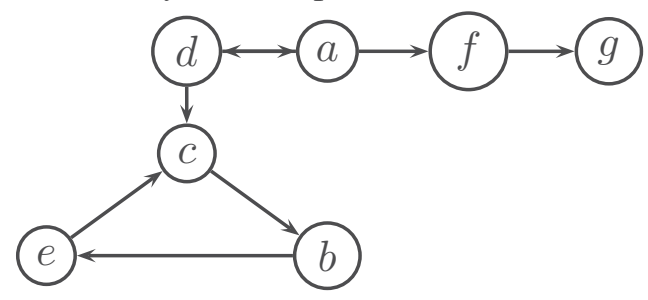

has five naive extensions: $\mathcal{E}_{1}=\{a, c, g\}, \mathcal{E}_{2}=\{d, e, f\}, \mathcal{E}_{3}=\{b, d, f\}, \mathcal{E}_{4}=\{a, e, g\}$, $\mathcal{E}_{5}=\{a, b, g\} ;$ one stable $\mathcal{E}_{3}$ and two preferred extensions $\mathcal{E}_{3}$ and $\mathcal{E}_{6}=\{a, g\}$.

It is worth recalling that stable extensions are naive (respectively preferred) extensions but the converses are not always true. Moreover, an argumentation framework may have no stable extensions.

Let us now define the plausible conclusions that may be drawn from a knowledge base $\Sigma$ by an argumentation system. The idea is to infer a formula $x$ from $\Sigma$ iff it is the conclusion of at least one argument in every extension of the system.

Definition 6 (Flat conclusions). Let $\mathcal{T}=(\operatorname{Arg}(\Sigma), \mathcal{R})$ be an argumentation system over a knowledge base $\Sigma$. The set of plausible conclusions of $\mathcal{T}$ is

$$
\operatorname{Output}(\mathcal{T})= \begin{cases}\{x \in \mathcal{L} \mid \forall \mathcal{E} \in \operatorname{Ext}(\mathcal{T}) \exists a \in \mathcal{E} \text { s.t. } \operatorname{Conc}(a)=x\} & \text { if } \operatorname{Ext}(\mathcal{T}) \neq \emptyset \\ \emptyset & \text { else }\end{cases}
$$


In [2] a comprehensive study has been made on the family of logics described in this section. It has been shown that when an argumentation system satisfies two key properties, then there is a full correspondence between the naive extensions of the system and the maximal consistent subbases of the knowledge base. More formally:

Postulates 1 (Closure under sub-arguments - Consistency). Let $\mathcal{T}=(\operatorname{Arg}(\Sigma), \mathcal{R})$ be an argumentation system over a knowledge base $\Sigma$. For all $\mathcal{E} \in \operatorname{Ext}(\mathcal{T})$,

- if $a \in \mathcal{E}$, then $\operatorname{Sub}(a) \subseteq \mathcal{E}$. We say that $\mathcal{T}$ is closed under sub-arguments.

- $\operatorname{Con} \operatorname{cs}(\mathcal{E})$ is consistent. We say that $\mathcal{T}$ satisfies consistency.

Theorem 1. [2] Let $\mathcal{T}=(\operatorname{Arg}(\Sigma), \mathcal{R})$ be an argumentation system over a knowledge base $\Sigma$. If $\mathcal{T}$ satisfies consistency and is closed under sub-arguments (under naive semantics), then:

- For all $\mathcal{E} \in \operatorname{Ext}_{n}(\mathcal{T})$, Base $(\mathcal{E}) \in \operatorname{Max}(\Sigma)$.

- For all $\mathcal{S} \in \operatorname{Max}(\Sigma), \operatorname{Arg}(\mathcal{S}) \in \operatorname{Ext}_{n}(\mathcal{T})$.

Let us now characterize the set of inferences that may be drawn from a knowledge base $\Sigma$ by any argumentation system under naive semantics. It coincides with the set of inferences that are drawn from the maximal consistent subsets of $\Sigma$.

Theorem 2. [2] Let $\mathcal{T}=(\operatorname{Arg}(\Sigma), \mathcal{R})$ be an argumentation system over a knowledge base $\Sigma$ such that $\mathcal{T}$ satisfies consistency and is closed under sub-arguments (under naive semantics).

$$
\operatorname{Output}(\mathcal{T})=\bigcap_{\mathcal{S}_{i} \in \operatorname{Max}(\Sigma)} \operatorname{CN}\left(\mathcal{S}_{i}\right)
$$

A similar study has been conducted for stable and preferred semantics. It has been shown that there are two families of attack relations. The first family leads to coherent systems (i.e., their stable extensions coincide with their preferred ones). Furthermore, stable extensions coincide with the naive ones. Such systems collapse then with the above discussed ones. The second family of relations allows choosing only some maximal consistent subbases of the knowledge base.

It is worth noticing that the paraconsistent logics defined from argumentation systems that use extension semantics restore consistency and return flat consequences.

\section{Logics Induced by Ranking Semantics}

Ranking semantics have been introduced in [1] as an alternative approach for evaluating arguments. Their basic idea is to rank arguments from the most to the less acceptable ones, instead of computing extensions. In what follows, we illustrate the approach with burden-based semantics (Bbs) introduced in [1]. Bbs assigns a burden number to every argument. The heavier the burden of an argument, the weaker its attacks. 
Definition 7 (Burden numbers). Let $\mathcal{T}=(\operatorname{Arg}(\Sigma), \mathcal{R})$ be an argumentation system, $i \in\{0,1, \ldots\}$, and $a \in \operatorname{Arg}(\Sigma)$. We denote by $\operatorname{Bur}_{\mathcal{T} i}(a)$ the burden number of a in the $i^{\text {th }}$ step, i.e.:

$$
\operatorname{Bur}_{i}(a)= \begin{cases}1 & \text { if } i=0 \\ 1+\sum_{b \in \operatorname{Att}(a)} 1 / \operatorname{Bur}_{i-1}(b) & \text { otherwise }\end{cases}
$$

where $\operatorname{Att}(a)=\{b \in \operatorname{Arg}(\Sigma) \mid(b, a) \in \mathcal{R}\}$.

By convention, if $\operatorname{Att}(a)=\emptyset$, then

$$
\sum_{b \in \operatorname{Att}(a)} 1 / \operatorname{Bur}_{i-1}(b)=0 .
$$

Let us illustrate this function in the following example.

Example 2. Assume the argumentation system depicted in the figure below.

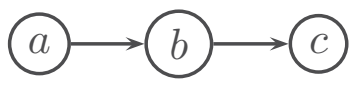

The burden numbers of each argument are summarized in the table below. Note that these numbers will not change beyond step 2.

\begin{tabular}{|c|c|c|c|}
\hline Step $i$ & $a$ & $b$ & $c$ \\
\hline 0 & 1 & 1 & 1 \\
1 & 1 & 2 & 2 \\
2 & 1 & 2 & 1.5 \\
$\vdots$ & $\vdots$ & $\vdots$ & $\vdots$ \\
\hline
\end{tabular}

Arguments are compared lexicographically on the basis of their burden numbers as follows:

Definition 8 (Bbs). The burden-based semantics Bbs transforms any argumentation system $\mathcal{T}=(\operatorname{Arg}(\Sigma), \mathcal{R})$ into the ranking $\operatorname{Bbs}(\mathcal{T})$ on $\operatorname{Arg}(\Sigma)$ such that $\forall a, b \in$ $\operatorname{Arg}(\Sigma),\langle a, b\rangle \in \operatorname{Bbs}(\mathcal{T})$ iff one of the two following cases holds:

, $\forall i \in\{0,1, \ldots\} \operatorname{Bur}_{i}(a)=\operatorname{Bur}_{i}(b)$;

, $\exists i \in\{0,1, \ldots\}, \operatorname{Bur}_{i}(a)<\operatorname{Bur}_{i}(b)$ and $\forall j \in\{0,1, \ldots, i-1\}, \operatorname{Bur}_{j}(a)=\operatorname{Bur}_{j}(b)$.

Intuitively, $\langle a, b\rangle \in \operatorname{Bbs}(\mathcal{T})$ means that $a$ is at least as acceptable as $b$. Let us see in an example how the semantics works.

Example 2 (Cont). According to Bbs, the argument $a$ is strictly more acceptable than $c$ which is itself strictly more acceptable than $b$.

The plausible conclusions of an argumentation system that uses ranking semantics are simply those supported by at least one argument. Note that a formula and its negation may both be plausible. This means that the approach tolerates inconsistency. More importantly, the conclusions are ranked from the most to the least plausible ones. A formula is ranked higher than another formula if it is supported by an argument which is more acceptable than any argument supporting the second formula. 
Definition 9 (Ranked conclusions). Let $\mathcal{T}=(\operatorname{Arg}(\Sigma), \mathcal{R})$ be an argumentation system over a knowledge base $\Sigma$. The output of $\mathcal{T}$ is the pair $\operatorname{Output}(\mathcal{T})=\langle\mathcal{C}, \preceq\rangle$ such that:

, $\mathcal{C}=\{\operatorname{Conc}(a) \mid a \in \operatorname{Arg}(\Sigma)\}$

- $x \preceq y$ iff $\exists a \in \operatorname{Arg}(\Sigma)$ such that $\operatorname{Conc}(a)=x$ and $\forall b \in \operatorname{Arg}(\Sigma)$ such that $\operatorname{Conc}(b)=$ $y,\langle a, b\rangle \in \operatorname{Bbs}(\mathcal{T})$.

Unlike certain well-known inconsistency-tolerating logics (like the the 3 and 4 valued ones $[4,6])$, the above logics satisfy the following crucial property: if the premises are consistent, the conclusions coincide with those of CN.

\section{Conclusion}

Argumentation is a natural approach for handling inconsistency. It is more akin to the way humans deal with inconsistency in everyday life. Indeed, generally people construct arguments pros and arguments cons opinions. As a consequence, the results of an argumentation approach are easier to grasp for an end-user. Yet, the approach is efficient since, as discussed in the paper, it is able to capture existing approaches (in the case of extension semantics) or even also to outperform some approaches on certain points (in the case of ranking semantics).

\section{References}

1. Amgoud, L., Ben-Naim, J.: Ranking-based semantics for argumentation frameworks. In: Liu, W., Subrahmanian, V.S., Wijsen, J. (eds.) SUM 2013. LNCS (LNAI), vol. 8078, pp. 134-147. Springer, Heidelberg (2013)

2. Amgoud, L., Besnard, P.: Logical limits of abstract argumentation frameworks. Journal of Applied Non-Classical Logics 23(3), 229-267 (2013)

3. Baroni, P., Giacomin, M., Guida, G.: Scc-recursiveness: A general schema for argumentation semantics. Artificial Intelligence Journal 168, 162-210 (2005)

4. Belnap, N.D.: A Useful Four-Valued Logic. In: Dunn, J., Epstein, G. (eds.) Modern Uses of Multiple-Valued Logic, pp. 7-37. Oriel Press (1977)

5. Cholvy, L.: Automated reasoning with merged contradictory information whose reliability depends on topics. In: Froidevaux, C., Kohlas, J. (eds.) ECSQARU 1995. LNCS, vol. 946, pp. 125-132. Springer, Heidelberg (1995)

6. D’Ottaviano, I., da Costa, N.: Sur un problème de Jaśkowski. In: Comptes Rendus de l'Académie des Sciences de Paris, vol. 270, pp. 1349-1353 (1970)

7. Dung, P., Mancarella, P., Toni, F.: Computing ideal skeptical argumentation. Artificial Intelligence Journal 171, 642-674 (2007)

8. Dung, P.M.: On the Acceptability of Arguments and its Fundamental Role in Non-Monotonic Reasoning, Logic Programming and n-Person Games. AIJ 77, 321-357 (1995)

9. Kleer, J.D.: Using crude probability estimates to guide diagnosis. Artificial Intelligence 45, 381-391 (1990)

10. Reiter, R.: A logic for default reasoning. Artificial Intelligence 13(1-2), 81-132 (1980)

11. Rescher, N., Manor, R.: On inference from inconsistent premises. Journal of Theory and Decision 1, 179-219 (1970)

12. Tarski, A.: On Some Fundamental Concepts of Metamathematics. In: Woodger, E.H. (ed.) Logic, Semantics, Metamathematics. Oxford Uni. Press (1956) 